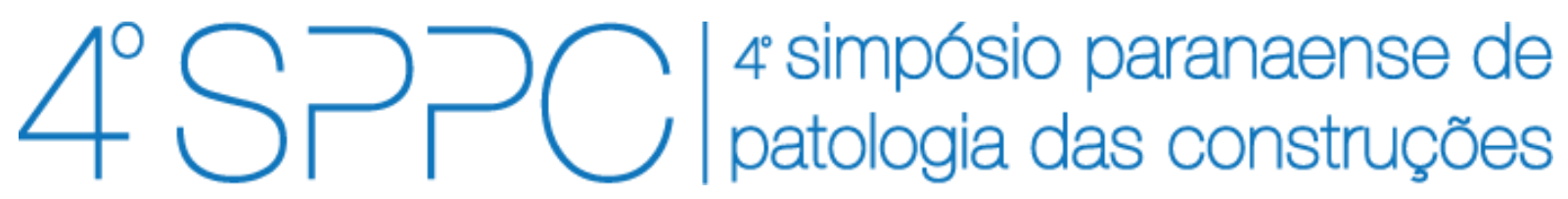

ISSN 2526-7248 artigo n. 4SPPC130, pp. 282-290, 2019

\title{
Comparativo entre cimento Portland e cimento supersulfatado submetidos ao ataque por sulfato de sódio: resistência mecânica e variação dimensional
}

Trentin, Priscila Ongaratto ${ }^{1}$; Magro, Isabel Cristina ${ }^{2}$; Souza, Laura Rorato Moraes Neubern ${ }^{3}$; Homrich, Jefferson Teixeira Olea 4; Angulski da Luz, Caroline ${ }^{5}$; Bonini, Janaína Sartori 6

${ }^{1}$ Mestranda, Universidade Tecnológica Federal do Paraná, priscilatrentin@hotmail.com

2 Estudante, Universidade Tecnológica Federal do Paraná, isabelmagro@alunos.utfpr.edu.br

${ }^{3}$ Estudante, Universidade Tecnológica Federal do Paraná, laurasouza@alunos.utfpr.edu.br

4 Doutorando, Universidade Federal do Paraná, eng.homrich@yahoo.com.br

${ }^{5}$ Professora, Universidade Tecnológica Federal do Paraná, angulski@hotmail.com

${ }^{6}$ PNPD, Universidade Tecnológica Federal do Paraná, janatoribon@yahoo.com.br

Resumo: Atualmente buscam-se materiais alternativos ao Cimento Portland (CP) pois ele possui um processo produtivo com alta emissão de $\mathrm{CO}_{2}$ ao meio ambiente. Um desses materiais é o cimento supersulfatado (CSS), que, além das vantagens ambientais, apresenta alta resistência à compressão, baixo calor de hidratação e boa durabilidade em ambientes agressivos. Devido a importância de estudar a durabiliade de novos materiais, este trabalho visou comparar o comportamento CSS em relação ao CP frente ao ataque por sulfato de sódio. Os resultados mostraram comportamento adequado do CSS, tanto em relação à resistência quanto à variação dimensional.

Palavras-chave: Cimento supersulfatado, Cimento Portland, Ataque por sulfatos.

Abstract: Currently, alternative materials are sought from Portland cement (PC) because it has a productive process with high $\mathrm{CO}_{2}$ emission to the environment. One of these materials is supersulfated cement (SSC), which, in addition to the environmental advantages, has high compressive strength, low heat of hydration and good durability in aggressive environments. Due to the importance of studying the durability of new materials, this work aimed to compare the SSC behavior in relation to PC against sodium sulfate attack. The results showed adequate behavior of the $\mathrm{SSC}$, both in relation to the resistance and the dimensional variation.

Keywords: Supersulfate cement, Portland cement, Sulphate attack. 
TRENTIN, P. O.; MAGRO. I.C.; SOUZA, L.R.M.N.; HOMRICH, J. T. O.; ANGULSKI DA LUZ, C.; BONINI, J.S. COMPARATIVO ENTRE CIMENTO PORTLAND E CIMENTO SUPERSULFATADO QUANDO SUBMETIDOS AO ATAQUE POR SULFATO DE SÓDIO: RESISTÊNCIA MECÂNICA E VARIAÇÃO DIMENSIONAL $4^{\circ}$ Simpósio Paranaense de Patologia das Construções (40 SPPC),

\section{Introdução}

Paralelamente à expansão da construção civil observam-se problemas relacionados à preservação de recursos naturais e à impactos ambientais. Analisando o panorama mundial, estima-se que as indústrias produtoras de cimento sejam responsáveis pelo lançamento de pelo menos $5 \%$ de todo gás carbônico $\left(\mathrm{CO}_{2}\right)$ na atmosfera, sendo esse lançamento advindo do processo de produção do clínquer [1]. Nesse contexto, a busca por materiais cimentícios ambiental e economicamente viáveis vem se destacando na construção civil. Uma das alternativas ao cimento Portland é o cimento supersulfatado (CSS), constituído por até $90 \%$ de escória de alto-forno (subproduto do beneficiamento do aço), até $20 \%$ de sulfato de cálcio e até $5 \%$ de ativador alcalino $[2,3]$.

Porém, sabe-se das barreiras impostas para implementar novos materiais na construção civil, tanto por parte dos profissionais da área quanto pelos consumidores. Ademais, a falta de normas técnicas para certificação de novos materiais prejudica esse processo [4].

Diante disso, justifica-se a necessidade de aprofundamento dos estudos em novos materiais de construção, como é o caso do CSS, pois, apesar da já conhecida redução do impacto ambiental advinda de seu processo produtivo, é importante estudar seu comportamento quanto à durabilidade.

De acordo com Fachinelli [5], a durabilidade do concreto está ligada aos seus componentes, ao ambiente de exposição da estrutura e às características físicoquímicas dos produtos gerados na hidratação do cimento.

Existem tanto causas físicas quanto causas químicas de deterioração do concreto. Uma forma de ocorrência simultânea de degradação física e química à que as estruturas de concreto estão expostas é o ataque por sulfatos, que define-se como uma ação deletéria que se manifesta por expansões, fissurações e redução gradual de resistência e massa [6, 7].

Diversos estudos tem buscado analisar o comportamento dos cimentos quanto ao ataque por sulfatos. Ding et al. [8], analisaram o comportamento de pastas de cimento Portland e de cimento Portland com $30 \%$ de escória de alto-forno em solução de sulfato de sódio durante o período de 365 dias. Os autores verificaram a descalcificação dos íons de cálcio do gel C-A-S-H (aluminossilicato de cálcio hidratado) e a formação de grande quantidade de etringita nas pastas produzidas apenas com cimento Portland.

Grounds, Nowell e Wilburn [3], estudaram o comportamento de prismas de CSS em diferentes temperaturas e taxas de umidade e verificaram maior resistência do CSS ao ataque por sulfato de sódio, quando comparado ao cimento referência. Entretanto, quando utilizado o sulfato de magnésio, os prismas de CSS foram decompostos em todas as condições ensaiadas.

Dessa forma, visando contribuir com os estudos de durabilidade de novos materiais, este trabalho tem por objetivo comparar o comportamento do cimento Portland e do cimento supersulfatado, quando submetidos ao ataque por sulfatos. 
TRENTIN, P. O.; MAGRO. I.C.; SOUZA, L.R.M.N.; HOMRICH, J. T. O.; ANGULSKI DA LUZ, C.; BONINI, J.S. COMPARATIVO ENTRE CIMENTO PORTLAND E CIMENTO SUPERSULFATADO QUANDO SUBMETIDOS AO ATAQUE POR SULFATO DE SÓDIO: RESISTÊNCIA MECÂNICA E VARIAÇÃO DIMENSIONAL $4^{\circ}$ Simpósio Paranaense de Patologia das Construções (40 SPPC),

\section{Materiais e métodos} artigo 4SPPC130, pp. 282 - 290, 2019. DOI: 10.4322/2526-7248.050

\subsection{Materiais}

\subsubsection{Escória granulada de alto-forno}

A escória utilizada tem massa específica de $2,83 \mathrm{~g} / \mathrm{cm}^{3}$ e superfície específica de $4460 \mathrm{~cm}^{2} / \mathrm{g}$. A composição química do material, especificada pelo fornecedor, está apresentada na Tab. 1.

Tabela 1: Composição química da escória granulada de alto-forno

\begin{tabular}{ccc}
\hline Elementos & Variação percentual & EN 15473:2010 \\
\hline $\mathrm{SiO}_{2}$ & 31,3 & - \\
$\mathrm{Fe}_{2} \mathrm{O}_{3}$ & 0,7 & - \\
$\mathrm{Al}_{2} \mathrm{O}_{3}$ & 13,3 & - \\
$\mathrm{CaO}$ & 42,8 & - \\
$\mathrm{SO}_{3}$ & 2,6 & - \\
$\mathrm{MgO}$ & 4,8 & - \\
$\mathrm{K}_{2} \mathrm{O}$ & 0,4 & - \\
$\mathrm{TiO}_{2}$ & 0,6 & - \\
L.O.I (Loss on Ignition/ perda ao fogo) & 2,6 & - \\
Adições inorgânicas do processo & 6 & - \\
\hline $\mathrm{CaO}+\mathrm{MgO}+\mathrm{SiO}_{2}$ & 78,9 & $\geq 66,7$ \\
\hline $\mathrm{CaO}+\mathrm{MgO} / \mathrm{SiO}_{2}$ & 1,52 & $\geq 1$ \\
\hline
\end{tabular}

De acordo com a classificação da norma EN 15743 [9], verifica-se que a escória de alto-forno pode ser utilizada na produção de CSS.

A análise de difratometria de raios-X, apresentada na Fig. 1, mostra uma caraterística amorfa da escória. Os picos relativos aos compostos de gipsita e gesso são oriundos da adição de gipsita durante o processo de moagem da própria escória.

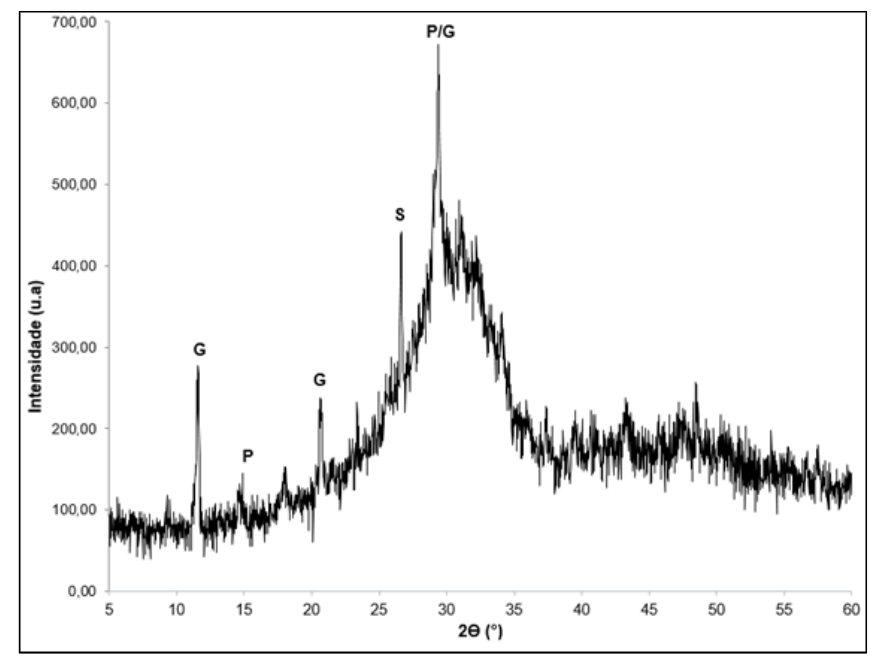

Figura 1: DRX da escória granulada de alto forno 
TRENTIN, P. O.; MAGRO. I.C.; SOUZA, L.R.M.N.; HOMRICH, J. T. O.; ANGULSKI DA LUZ, C.; BONINI, J.S. COMPARATIVO ENTRE CIMENTO PORTLAND E CIMENTO SUPERSULFATADO QUANDO SUBMETIDOS AO ATAQUE POR SULFATO DE SÓDIO: RESISTÊNCIA MECÂNICA E VARIAÇÃO DIMENSIONAL $4^{\circ}$ Simpósio Paranaense de Patologia das Construções (40 SPPC),

2.1.2 Sulfato de cálcio artigo 4SPPC130, pp. 282 - 290, 2019. DOI: 10.4322/2526-7248.050

Para a produção do CSS, como fonte de sulfato de cálcio foi utilizada a gipsita, que passou por um processo de moagem em moinho de bolas, peneiramento em peneira $150 \mu \mathrm{m}$ e calcinação a temperatura de $650{ }^{\circ} \mathrm{C}$ por 60 minutos. A transformação da gipsita da fase hidratada para anidra pode ser observada na Fig. 2.

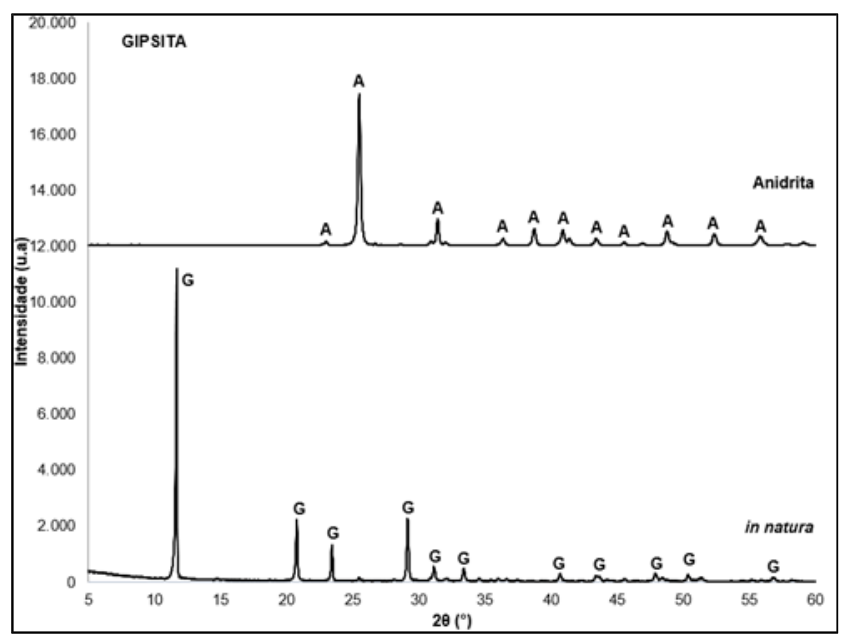

Figura 2: DRX da gipsita in natura e anidra

\subsubsection{Cimento Portland V - ARI}

O Cimento Portland tipo $V$ de alta resistência inicial, da marca Itambé, foi utilizado como ativador alcalino para produção do cimento supersulfatado.

\subsubsection{Cimento Portland V - ARI RS}

O Cimento Portland tipo $V$ de alta resistência inicial e resistente a sulfatos, da Votorantim Cimentos, foi utilizado como composição padrão para a comparação com o cimento supersulfatado. Segundo o fabricante, esse cimento tem massa específica de $2,97 \mathrm{~g} / \mathrm{cm}^{3}$ e superfície específica de $5027 \mathrm{~cm}^{2} / \mathrm{g}$. A composição química do cimento, também fornecida pela empresa fabricante, é apresentada na Tab. 2.

Tabela 2: Composição química do CP V-ARI RS

\begin{tabular}{cc}
\hline Caracterização química & Teores \\
\hline Perda ao fogo & $4,4 \%$ \\
$\mathrm{MgO}$ & $5,6 \%$ \\
$\mathrm{SO}_{3}$ & $2,9 \%$ \\
Resíduo insolúvel & $13,4 \%$ \\
\hline
\end{tabular}


TRENTIN, P. O.; MAGRO. I.C.; SOUZA, L.R.M.N.; HOMRICH, J. T. O.; ANGULSKI DA LUZ, C.; BONINI, J.S. COMPARATIVO ENTRE CIMENTO PORTLAND E CIMENTO SUPERSULFATADO QUANDO SUBMETIDOS AO ATAQUE POR SULFATO DE SÓDIO: RESISTÊNCIA MECÂNICA E VARIAÇÃO DIMENSIONAL $4^{\circ}$ Simpósio Paranaense de Patologia das Construções (40 SPPC),

\subsection{Métodos} artigo 4SPPC130, pp. 282 - 290, 2019. DOI: 10.4322/2526-7248.050

\subsubsection{Formulação dos cimentos}

As pastas de CSS e de CP V-ARI RS foram preparadas com relação água/ cimento (a/c) 0,45. A Tab. 3 a seguir relacionada apresenta as proporções dos materiais utilizados.

Tabela 3: Quantidade de material das pastas (em g)

\begin{tabular}{cccccc}
\hline Cimento & Escória & Anidrita & CP V-ARI & CP V-ARI RS & Água \\
\hline CP V-ARI RS & - & - & - & 100 & 45 \\
CSS 90.10 G & 85,5 & 9,5 & 5 & & 45
\end{tabular}

\subsubsection{Solução de ataque}

A solução de sulfato de sódio foi preparada a fim de simular um ambiente agressivo com alta concentração de sulfatos. A preparação consistiu na dissolução de 50 gramas de sulfato de sódio anidro $\left(\mathrm{Na}_{2} \mathrm{SO}_{4}\right)$, granulado, com pureza de $99 \%$, distribuído pela empresa Alphatec (P.A), em um litro de água destilada, o que corresponde à concentração de $50 \mathrm{~g} / \mathrm{L}$.

\subsubsection{Resistência à compressão}

Para o ensaio de resistência à compressão foram moldados três corpos de prova de $30 \times 30 \times 30 \mathrm{~mm}$ para cada tipo de cimento utilizado. Após 48 horas da moldagem foi realizada a desmoldagem, sendo os corpos de prova colocados em cura submersa de água saturada com cal até completarem a idade de 7 dias, quando foram submetidos ao ataque por sulfatos.

O ensaio foi realizado aos 7 dias, antes do início do ataque pelo $\mathrm{Na}_{2} \mathrm{SO}_{4}$ e após 49 dias de contato com a solução.

\subsubsection{Análise da variação dimensional}

Para análise de variação dimensional foi utilizada a metodologia NIST Test, conforme realizado por Aleksic [10], que consiste na moldagem de sete primas retangulares com dimensões de 10×10×60 mm e aplicação de resina epóxi nas extremidades e laterais dos corpos de prova para estabilizar o pino de medição e evitar a entrada de sulfatos nessa região. Da mesma forma como ocorreu no ensaio de resistência mecânica, os corpos de prova foram curados em água saturada com cal e, quando completados 7 dias de idade, os mesmos foram submetidos ao ataque por sulfato, ficando submersos em solução de sulfato de sódio.

Com a utilização de um relógio comparador de comprimento, que pode ser observado na Fig. 3, foram realizadas medições diárias até completadas duas semanas de análise. Após esse período, as medições foram realizadas semanalmente, sendo a 
TRENTIN, P. O.; MAGRO. I.C.; SOUZA, L.R.M.N.; HOMRICH, J. T. O.; ANGULSKI DA LUZ, C.; BONINI, J.S. COMPARATIVO ENTRE CIMENTO PORTLAND E CIMENTO SUPERSULFATADO QUANDO SUBMETIDOS AO ATAQUE POR SULFATO DE SÓDIO:

RESISTÊNCIA MECÂNICA E VARIAÇÃO DIMENSIONAL $4^{\circ}$ Simpósio Paranaense de Patologia das Construções (40 SPPC) artigo 4SPPC130, pp. 282 - 290, 2019. DOI: 10.4322/2526-7248.050

solução de $\mathrm{Na}_{2} \mathrm{SO}_{4}$ substituída depois da realização de cada medição. A variação dimensional de cada corpo de prova foi obtida pela diferença entre o valor medido na idade correspondente e a leitura inicial do corpo de prova, dividida pelo seu comprimento efetivo e multiplicada por 100.
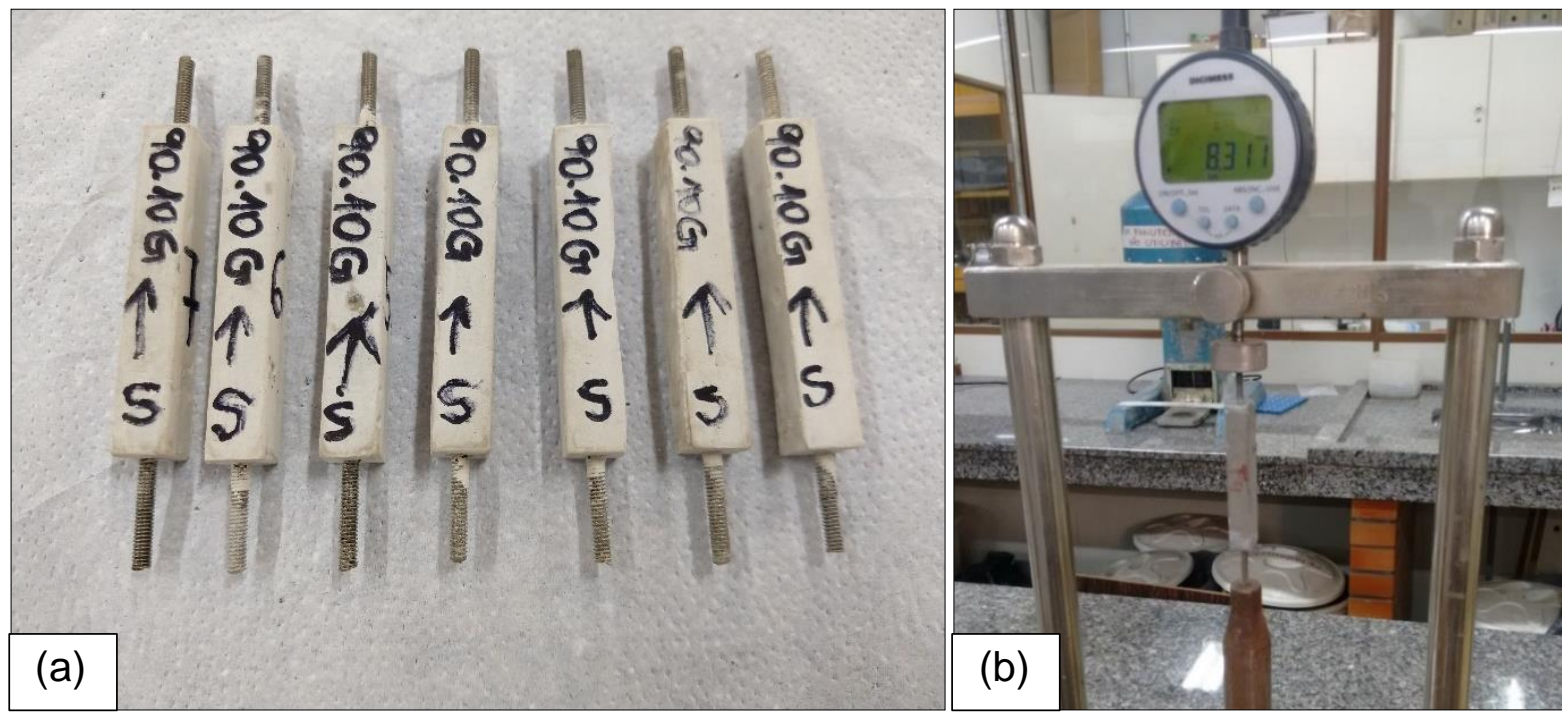

Figura 3: (a) Corpos de prova de pasta antes da exposição ao ataque por $\mathrm{Na}_{2} \mathrm{SO}_{4}$; (b) Verificação da variação dimensional no comparador de comprimento.

\section{Resultados}

A Fig. 4 apresenta os resultados de resistência à compressão quando da exposição dos corpos de prova ao ataque de $\mathrm{Na}_{2} \mathrm{SO}_{4}$. Como pode ser observado, o CSS apresenta menor resistência em comparação com o CP V-ARI RS, tanto aos 7 dias, quando em cura em água com cal, quanto aos 49 dias, quando imersos em solução de sulfato de sódio.

Aos 7 dias a resistência do CSS foi 20,5\% inferior ao cimento Portland e, aos 49 dias, foi $23,0 \%$ inferior. Com o tratamento estatístico dos dados no programa STATISTICA 8.0, adotando-se um índice de significância de $5 \%$, foi realizado o Teste Tukey, onde verificou-se que os valores são estatisticamente diferentes entre si. Apesar da menor resistência apresentada pelo CSS, o ganho de resistência desse cimento acompanhou o aumento de resistência apresentado pelo CP V-ARI RS.

Gruskovnjak et al. [2], estudaram sobre o desenvolvimento de resistência de pastas de cimento supersulfatado com $85 \%$ de escória, $15 \%$ de anidrita e $0,5 \%$ de $\mathrm{KOH}$ e verificaram que o desenvolvimento de resistência mecânica inicial é mais rápido quando utiliza-se escórias de alta reatividade, como é o caso da escória utilizada neste trabalho. Segundo os autores, o ganho de resistência deve-se à formação de agulhas de etringita, que preenchem a porosidade da matriz. 
TRENTIN, P. O.; MAGRO. I.C.; SOUZA, L.R.M.N.; HOMRICH, J. T. O.; ANGULSKI DA LUZ, C.; BONINI, J.S. COMPARATIVO ENTRE CIMENTO PORTLAND E CIMENTO SUPERSULFATADO QUANDO SUBMETIDOS AO ATAQUE POR SULFATO DE SÓDIO: RESISTÊNCIA MECÂNICA E VARIAÇÃO DIMENSIONAL $4^{\circ}$ Simpósio Paranaense de Patologia das Construções (40 SPPC), artigo 4SPPC130, pp. $282-290$, 2019. DOI: $10.4322 / 2526-7248.050$

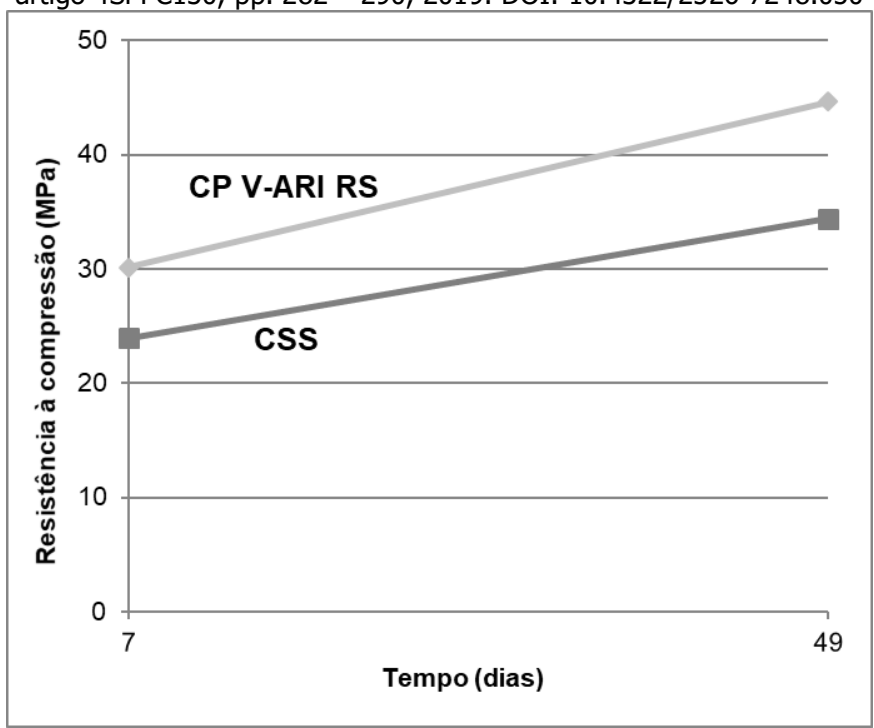

Figura 4: Resistência mecânica dos corpos de prova expostos ao sulfato de sódio

A Fig. 5 apresenta os resultados da variação dimensional dos corpos de prova submetidos ao ataque por $\mathrm{Na}_{2} \mathrm{SO}_{4}$ durante 56 dias. Considerando que o limite de expansão estabelecido pelo NIST Test é de $0,10 \%$ em 56 dias, pode-se observar na que o cimento Portland ultrapassa esse limite aos 42 dias de ataque. Já no caso do CSS observou-se um comportamento de retração desde o início das análises.

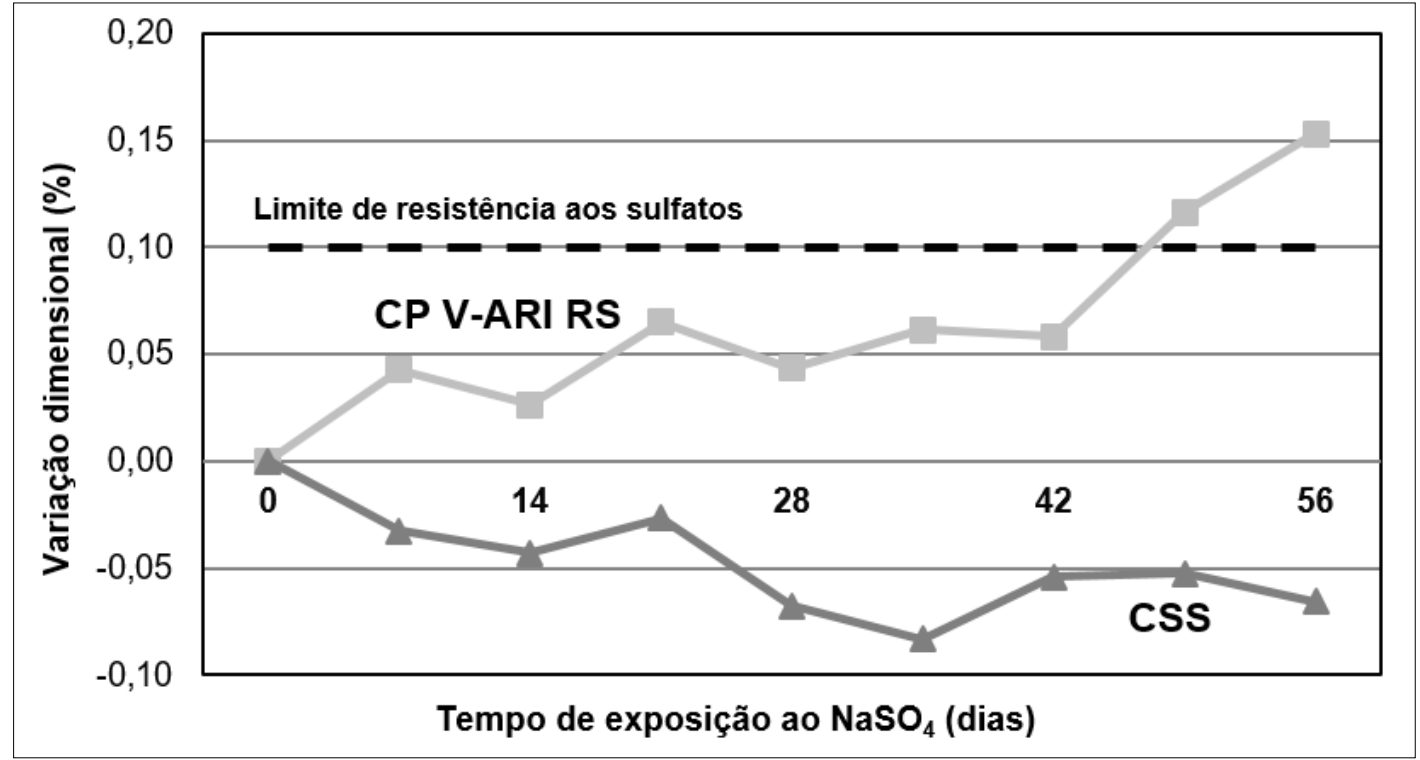

Figura 5: Variação dimensional dos corpos de prova expostos ao sulfato de sódio 
TRENTIN, P. O.; MAGRO. I.C.; SOUZA, L.R.M.N.; HOMRICH, J. T. O.; ANGULSKI DA LUZ, C.; BONINI, J.S. COMPARATIVO ENTRE CIMENTO PORTLAND E CIMENTO SUPERSULFATADO QUANDO SUBMETIDOS AO ATAQUE POR SULFATO DE SÓDIO: RESISTÊNCIA MECÂNICA E VARIAÇÃO DIMENSIONAL $4^{\circ}$ Simpósio Paranaense de Patologia das Construções (40 SPPC), artigo 4SPPC130, pp. 282 - 290, 2019. DOI: 10.4322/2526-7248.050

O melhor comportamento apresentado pelo CSS quanto a variação dimensional corroboram com alguns autores que avaliaram o desempenho desse cimento quanto ao ataque por sulfato de sódio. Segundo Pinto [4], o bom desempenho do CSS frente ao sulfato de sódio deve-se à menor disponibilidade de hidratos (aluminatos e portlandita) passíveis de reagir com os íons sulfatos. Apesar disso, uma retração excessiva também pode ser nociva para as pastas de cimento, pois a retração causa fissuração das pastas.

\section{Conclusão}

Este estudo buscou comparar o comportamento do cimento Portland, tradicionalmente utilizado em situações de ataque por sulfato e do cimento supersulfatado, quando submetidos ao ataque por sulfato de sódio, e após os ensaios realizados, é possível concluir:

- Quanto à resistência mecânica, obteve-se melhores resultados com o CP VARI RS; apesar disso, o ganho de resistência do CSS acompanhou o cimento Portland e as resistências obtidas permitem a aplicação desse cimento para diversas finalidades.

- Em relação à variação dimensional, nota-se uma significativa diferença entre o comportamento dos dois tipos de cimentos. Enquanto o CSS apresentou retração durante todo o período analisado, O CP V-ARI RS expandiu, ultrapassando o limite de expansão estabelecido pela metodologia NIST Test aos 42 dias de ensaio.

- A retração apresentada pelo CSS acorda com os resultados da literatura, que apontam bom comportamento desse cimento quando exposto ao ataque por sulfato de sódio. Apesar disso, uma retração excessiva pode causar fissuração, comprometendo a durabilidade do material.

- De maneira geral, verificou-se um comportamento adequado do CSS quando exposto ao ataque por $\mathrm{Na}_{2} \mathrm{SO}_{4}$ mas, recomenda-se que outros estudos sejam realizados, especialmente para avaliar o comportamento desse cimento à longo prazo.

\section{Agradecimentos}

À Coordenação de Aperfeiçoamento de Pessoal de Nível Superior (CAPES) pela concessão de bolsas de estudos que permitiram o desenvolvimento deste estudo. 
TRENTIN, P. O.; MAGRO. I.C.; SOUZA, L.R.M.N.; HOMRICH, J. T. O.; ANGULSKI DA LUZ, C.; BONINI, J.S. COMPARATIVO ENTRE CIMENTO PORTLAND E CIMENTO SUPERSULFATADO QUANDO SUBMETIDOS AO ATAQUE POR SULFATO DE SÓDIO: RESISTÊNCIA MECÂNICA E VARIAÇÃO DIMENSIONAL $4^{\circ}$ Simpósio Paranaense de Patologia das Construções (40 SPPC),

\section{Referências} artigo 4SPPC130, pp. 282 - 290, 2019. DOI: 10.4322/2526-7248.050

[1] Lopes, D. F. (2011) Créditos de carbono na indústria do cimento. Trabalho de conclusão de curso, Universidade Federal do Rio Grande do Sul-UFRGS, Porto Alegre, Brasil.

[2] Gruskovnjak, A.; Lothenbach, B.; Winnefeld, F.; Figi, R.; Ko, S.C.; Adler, M.; Mäder, U. (2008) Hydration mechanisms of super sulphated slag cement, Cement and Concrete Research, 38:983-992.

[3] Grounds, T.; Nowell, D.V.; Wilburn, F.W. (2003) Resistance of supersulfated cement to strong sulfate solutions, Journal of Thermal Analysis and Calorimetry, 72:181-190.

[4] Pinto, S.R. (2019) Durabilidade de compósitos com cimento supersulfatado e de cimentos Portland: ataque por sulfatos, carbonatação e penetração de cloretos. Dissertação (mestrado) - Universidade federal do Paraná-UFPR, Curitiba, Brasil.

[5] Faquinelli, C.R. (2010) Estudo da reatividade de agregados contendo sulfetos em concretos e argamassas. Dissertação (mestrado), Universidade Federal de Minas Gerais-UFMG, Belo Horizonte, Brasil.

[6] Mehta, P.K.; Monteiro, P.J.M. (2008) Concreto: estrutura, propriedades e materiais. 1. ed. São Paulo, Brasil.

[7] Souza, R.B. (2006) Suscetibilidade de pastas de cimento ao ataque por sulfatos - Método de ensaio acelerado. Dissertação (mestrado), Universidade de São Paulo-USP, São Paulo, Brasil.

[8] Ding, Q.; Yang, J.; Hou, D.; Zhang, G. (2018) Insight on the mechanism of sulfate attacking on the cement paste with granulated blast furnace slag: An experimental and molecular dynamics study, Construction and Building Materials, 169: 601611.

[9] EN 15743 (2010) Supersulfated cement - Composition, specifications and conformity criteria. European Committee for Stardardization, Brussels, Belgium.

[10] Aleksic, M. (2010) Development and Standardization of the NIST Rapid Sulphate Resistance Test. 2010. Dissertation (Master) - University of Toronto, Toronto, Canada. 